BMJ Open Diabetes Research \& Care

\section{Glycemic efficacy and safety of the SGLT2 inhibitor ertugliflozin in patients with type 2 diabetes and stage 3 chronic kidney disease: an analysis from the VERTIS CV randomized trial}

To cite: Dagogo-Jack S Pratley RE, Cherney DZI, et al. Glycemic efficacy and safety of the SGLT2 inhibitor ertugliflozin in patients with type 2 diabetes and stage 3 chronic kidney disease: an analysis from the VERTIS CV randomized trial. BMJ Open Diab Res Care 2021;9:e002484. doi:10.1136/ bmjdrc-2021-002484

\section{- Additional supplemental material is published online only. To view, please visit the journal online (http://dx.doi. org/10.1136/bmjdrc-2021- 002484).}

Received 19 July 2021 Accepted 11 September 2021

Check for updates

(c) Author(s) (or their employer(s)) 2021. Re-use permitted under CC BY-NC. No commercial re-use. See rights and permissions. Published by BMJ.

For numbered affiliations see end of article.

Correspondence to Dr Samuel Dagogo-Jack; sdj@uthsc.edu

\section{ABSTRACT}

Introduction Here we report the glycemic efficacy and safety of ertugliflozin in patients in the VERTIS CV cardiovascular outcome trial with chronic kidney disease (CKD) stage 3.

Research design and methods Prespecified and posthoc analyses were performed in patients with an estimated glomerular filtration rate (eGFR) $30-<60 \mathrm{~mL} / \mathrm{min} / 1.73 \mathrm{~m}^{2}$ at screening. The primary endpoint was glycemic efficacy at week 18. Longer term glycemic efficacy and changes in body weight, systolic blood pressure (SBP), and eGFR were also evaluated.

Results Among 8246 patients in VERTIS CV, 1776 patients had CKD stage 3; 1319 patients had CKD stage 3A (eGFR $45-<60 \mathrm{~mL} / \mathrm{min} / 1.73 \mathrm{~m}^{2}$ ); 457 patients had CKD stage 3B (eGFR $30-<45 \mathrm{~mL} / \mathrm{min} / 1.73 \mathrm{~m}^{2}$ ). Week 18 least squares (LS)-mean $(95 \% \mathrm{Cl})$ placebo-adjusted changes from baseline in glycated hemoglobin (HbA1c) for $5 \mathrm{mg}$ and $15 \mathrm{mg}$ ertugliflozin were $-0.27 \%(-0.37 \%$ to $-0.17 \%)$ and $-0.28 \%(-0.38 \%$ to $-0.17 \%)$, respectively, for CKD stage 3 overall and $-0.27 \%(-0.38 \%$ to $-0.15 \%)$ and $-0.31 \%(-0.43 \%$ to $-0.19 \%)$, respectively, for CKD stage $3 \mathrm{~A}$ (all $\mathrm{p}<0.001$ ). For CKD stage $3 \mathrm{~B}$, the reduction in $\mathrm{HbA} 1 \mathrm{c}$ for $5 \mathrm{mg}$ ertugliflozin was $-0.28 \%(-0.47 \%$ to $-0.08 \%)$ $(p=0.006)$ and for $15 \mathrm{mg}$ ertugliflozin was $-0.19 \%$ $(-0.39 \%$ to $0.01 \%)(p=0.064)$. LS-mean placebo-adjusted reductions in body weight (range: -1.32 to $-1.95 \mathrm{~kg}$ ) and SBP (range: -2.42 to $-3.41 \mathrm{~mm} \mathrm{Hg}$ ) were observed across CKD stage 3 categories with ertugliflozin. After an initial dip, eGFR remained above or near baseline with ertugliflozin treatment. The incidence of overall adverse events (AEs), symptomatic hypoglycemia, hypovolemia, and kidney-related AEs did not differ between ertugliflozin and placebo across CKD stage 3 subgroups.

Conclusions In VERTIS CV patients with CKD stage 3A, ertugliflozin resulted in reductions in $\mathrm{HbA} 1 \mathrm{c}$, body weight and SBP, maintenance of eGFR, and was generally well tolerated. Results in the CKD stage 3B subgroup were generally similar except for an attenuated $\mathrm{HbA1c}$ response with the $15 \mathrm{mg}$ dose.

Trial registration number NCT01986881.

\section{Significance of this study}

What is already known about this subject?

- Patients with type 2 diabetes mellitus (T2DM) and chronic kidney disease (CKD) are at increased risk of further progression of CKD.

- The glycemic efficacy of sodium-glucose cotransporter 2 (SGLT2) inhibitors is dependent on glomerular filtration and is attenuated in patients with CKD stage 3 (estimated glomerular filtration rate (eGFR) $30-<60 \mathrm{~mL} / \mathrm{min} / 1.73 \mathrm{~m}^{2}$ ); however, independent of glycemic efficacy, members of the SGLT2 inhibitor class have been shown to slow the deterioration of kidney function in this patient population.

What are the new findings?

- This secondary analysis of the ertugliflozin cardiovascular outcome study, VERTIS CV, explored the glycemic efficacy and safety of ertugliflozin, compared with placebo, in patients with CKD stage 3.

- Treatment with ertugliflozin 5 and $15 \mathrm{mg}$ doses, compared with placebo, resulted in modest reductions in glycated hemoglobin from baseline, which were maintained long term; reductions in body weight and systolic blood pressure were similar to those observed in previous analyses of patients with preserved kidney function.

- The safety profile of ertugliflozin in VERTIS CV patients with CKD stage 3 was consistent with previous assessments of ertugliflozin safety; ertugliflozin, compared with placebo, slowed the decrease of eGFR over time.

How might these results change the focus of research or clinical practice?

- The present analyses add to the growing body of evidence on the positive benefit/risk profile of the SGLT2 inhibitor class in patients with T2DM and CKD stage 3. 


\section{INTRODUCTION}

Diabetes mellitus is a common cause of chronic kidney disease $(\mathrm{CKD}) .{ }^{1}$ A recent analysis of National Health and Nutrition Examination Survey (NHANES) data showed that among US adults with diabetes, $28.1 \%$ had concomitant CKD (defined as an estimated glomerular filtration rate $(\mathrm{eGFR})<60 \mathrm{~mL} / \mathrm{min} / 1.73 \mathrm{~m}^{2}$ or a urinary albumincreatinine ratio (UACR) $\geq 30 \mathrm{mg} / \mathrm{g}$ ) ${ }^{2}$ NHANES data have also shown a $\sim 5$-fold increase in the incidence of CKD in patients with diabetes versus patients without diabetes. ${ }^{3}$ Furthermore, people with CKD are at high risk of further deterioration of kidney function over time and at risk of cardiovascular (CV) events including myocardial infarction, stroke, and heart failure. According to the US Renal Data System 2020 Annual Data Report, heart failure and acute myocardial infarction were about four times as common and stroke/transient ischemic attack was more than twice as common in patients with CKD versus those without CKD. ${ }^{4}$ Treatment of these inter-related conditions has become a prominent focus of the treatment of people with type 2 diabetes mellitus (T2DM) and CKD. ${ }^{5}$

The glycemic efficacy of sodium-glucose cotransporter 2 (SGLT2) inhibitors is dependent on glomerular filtration, and glycemic efficacy is attenuated in patients with CKD stage 3 (eGFR $30-<60 \mathrm{~mL} / \mathrm{min} / 1.73 \mathrm{~m}^{2}$ ). However, SGLT2 inhibitors have beneficial CV and kidney protective effects in patients with T2DM that are related to glycemic control and that are independent of glycemic control. ${ }^{6-8}$ Based on the results from multiple outcome trials that have demonstrated the beneficial effects of SGLT2 inhibitors on CV and kidney endpoints, including in patients with CKD stage 3, society guidelines recommend the addition of an SGLT2 inhibitor for patients with T2DM and established CKD (eGFR $30-\leq 60 \mathrm{~mL}$ / $\min / 1.73 \mathrm{~m}^{2}$ or UACR $>30 \mathrm{mg} / \mathrm{g}$ ) to reduce the risk of CKD progression and future CV events, including hospitalization for heart failure (HHF) ${ }^{9-11}$

Ertugliflozin is an SGLT2 inhibitor approved for use in adults with T2DM as an adjunct to diet and exercise to improve glycemic control. ${ }^{12}{ }^{13}$ VERTIS CV was the CV outcome trial for ertugliflozin, conducted in patients with T2DM and atherosclerotic cardiovascular disease (ASCVD), which assessed the CV safety of ertugliflozin. ${ }^{14}{ }^{15}$ Enrollment criteria in VERTIS CV allowed for the entry of patients with an eGFR as low as $30 \mathrm{~mL} /$ $\mathrm{min} / 1.73 \mathrm{~m}^{2}$ at screening, providing an opportunity for a characterization of glycemic efficacy, CV outcomes, and safety across a spectrum of kidney function. The present analyses assessed the glycemic efficacy and safety of ertugliflozin in the subgroup of VERTIS CV patients with CKD stage 3 at trial entry.

\section{MATERIALS AND METHODS}

\section{Study design}

Glycemic efficacy and safety were assessed in the subset of patients with baseline CKD stage 3 who participated in VERTIS CV. VERTIS CV was a prospective, randomized, double-blind, placebo-controlled, parallel-group, multicenter trial conducted in patients with T2DM and established ASCVD comparing ertugliflozin versus placebo. ${ }^{14}$ 15 Eligible patients were randomly assigned $(1: 1: 1)$ to once-daily ertugliflozin $5 \mathrm{mg}$ or $15 \mathrm{mg}$ or placebo in addition to background standard-of-care treatment. During the initial 18 weeks after randomization, patients' background antihyperglycemic regimen was to remain stable unless glycemic rescue was required, or the patient experienced clinically significant hypoglycemia. This design facilitated the assessment of glycemic efficacy and other metabolic parameters in those patients with CKD stage 3, compared with placebo, without the confounding influence of changes in background antihyperglycemic therapy during the first 18 weeks.

\section{Patient population}

Detailed inclusion and exclusion criteria for the trial have been published previously. ${ }^{14}{ }^{15}$ Adult patients ( $\geq 40$ years of age) with T2DM (glycated hemoglobin (HbA1c) $7.0 \%-10.5 \%$, inclusive) and stable, established ASCVD of the coronary, cerebrovascular, and/ or peripheral arterial systems were included. Key exclusion criteria were type 1 diabetes, history of ketoacidosis, and eGFR $<30 \mathrm{~mL} / \mathrm{min} / 1.73 \mathrm{~m}^{2}$, calculated using the four-variable Modification of Diet in Renal Disease study equation. ${ }^{16}$ For the present subgroup analyses, patients with CKD stage 3 were classified based on an eGFR of $30-<60 \mathrm{~mL} / \mathrm{min} / 1.73 \mathrm{~m}^{2}$ at baseline; patients with CKD stage 3A based on an eGFR of $45-<60 \mathrm{~mL} /$ $\min / 1.73 \mathrm{~m}^{2}$ at baseline; and patients with CKD stage $3 \mathrm{~B}$ based on an eGFR of $30-<45 \mathrm{~mL} / \mathrm{min} / 1.73 \mathrm{~m}^{2}$ at baseline.

\section{Efficacy outcomes and analysis}

Subgroup analyses of glycemic efficacy outcomes in patients with CKD stage $3 \mathrm{~A}$ were prespecified in the VERTIS CV statistical analysis plan. The prespecified glycemic efficacy outcomes were change from baseline to week 18 in HbAlc and fasting plasma glucose (FPG), and the proportion of patients with HbAlc $<7.0 \%$ at week 18. Post-hoc analyses of change from baseline to week 18 in body weight and systolic blood pressure (SBP) were also conducted. Analyses of change over time in HbA1c at weeks 18, 52, 104, 156, and 208 in patients with CKD stage 3 were prespecified. The subgroup analyses of the same efficacy outcomes in patients with CKD stage 3 and CKD stage $3 \mathrm{~B}$ were post-hoc analyses, conducted using the same statistical methodology as the prespecified analyses. Analyses included those patients in the respective subgroup who were randomized, received at least one dose of study medication (ertugliflozin or placebo), and had at least one assessment at or after baseline. Efficacy data reported for week 18 endpoints were censored after initiation of any glycemic rescue therapy if the rescue occurred prior to week 18. Long-term assessments of 
efficacy included data regardless of changes in background antihyperglycemic medications.

Least squares (LS)-mean change from baseline to week 18 in efficacy outcomes with 95\% CIs was calculated using a constrained longitudinal data analysis (cLDA) model with fixed effects for treatment, time (categorical), baseline eGFR, and the interaction of time-by-treatment. Change over time in HbAlc at weeks 18, 52, 104, 156, and 208 was calculated using an analysis of covariance model adjusted for baseline HbA1c, treatment, time, subgroup, treatment-bysubgroup interaction, and treatment-by-subgroupby-time interaction. Adjusted ORs for patients with HbAlc $<7.0 \%$ at week 18 with $95 \%$ CIs were calculated using a logistic regression model fitted with fixed effects for treatment and covariates for baseline HbA1c and baseline eGFR. Missing data for HbA1c in the logistic regression model were multiply imputed using predicted values from the cLDA model of the primary analysis. In the overall subgroup of patients with CKD stage 3, week $18 \mathrm{HbA1c}$ values were missing in 120 of $617(19.4 \%), 102$ of $559(18.2 \%)$, and 130 of $597(21.8 \%)$ patients in the full analysis set for the ertugliflozin $5 \mathrm{mg}, 15 \mathrm{mg}$, and placebo treatment groups, respectively. Nominal $\mathrm{p}$ values (unadjusted for multiplicity) were calculated.

\section{Safety outcomes and analysis}

The safety analysis population included all patients with CKD stage 3 who had undergone randomization and received at least one dose of study medication (ertugliflozin or placebo). Safety analyses included data collected over the entire duration of the VERTIS CV trial (not limited to the week 18 efficacy endpoint). Safety analyses included adverse events (AEs) collected from randomization to 14 days after the final dose of study medication and 2 days after the final dose of study medication for laboratory endpoints. Safety was analyzed separately for patients with CKD stage 3, CKD stage 3A, and CKD stage 3B; the analyses were designed to compare treatment groups within each subgroup. Assessments included AEs, serious AEs (SAEs), discontinuations due to AEs, deaths, vital signs, and laboratory evaluations. The Medical Dictionary for Regulatory Activities (MedDRA; V.22.1) was used to classify AEs with respect to system organ class and preferred term. Kidney-related AEs were identified using the Standardised MedDRA Query of 'Acute renal failure'. Adjudication of potential kidney events was performed by an independent, blinded committee to assess causality relative to study medication as 'Very likely', 'Probable', 'Possible', 'Doubtful', and 'Not related'. Potential kidney events were identified by systematically searching AEs, SAEs, and predefined limits of change (doubling of serum creatinine from baseline or $\geq 50 \%$ decrease from baseline eGFR). Prespecified AEs of special clinical interest included AEs related to urinary tract infection (UTI), genital mycotic infection (GMI), symptomatic hypoglycemia, and hypovolemia.

\section{RESULTS}

\section{Patient disposition and baseline characteristics}

In VERTIS CV, a total of 8246 patients with T2DM and ASCVD underwent randomization; 8238 patients received at least one dose of ertugliflozin or placebo. ${ }^{1415}$ Of the 8246 patients randomized, 1776 patients with CKD stage 3 at baseline were identified based on an eGFR of $30-<60 \mathrm{~mL} / \mathrm{min} / 1.73 \mathrm{~m}^{2}$, comprising 1319 patients with CKD stage 3A (eGFR $45-<60 \mathrm{~mL}$ / $\min / 1.73 \mathrm{~m}^{2}$ ) and 457 patients with CKD stage 3B (eGFR $30-<45 \mathrm{~mL} / \mathrm{min} / 1.73 \mathrm{~m}^{2}$ ).

Baseline demographic and clinical characteristics were well balanced across treatment groups in the subgroup of patients with CKD stage 3 (table 1 ). In the overall population of patients with CKD stage 3 , the majority of patients were white $(86.1 \%)$ and male $(64.3 \%)$; mean baseline age $( \pm \mathrm{SD})$ was $68.1 \pm 7.6$ years; mean duration of T2DM was $15.7 \pm 8.9$ years; mean $\mathrm{HbAlc}$ was $8.2 \% \pm 0.9 \%$; and mean eGFR was $49.3 \pm 7.5 \mathrm{~mL} / \mathrm{min} / 1.73 \mathrm{~m}^{2}$.

Baseline characteristics were also similar across treatment groups in the CKD stage $3 \mathrm{~A}$ and CKD stage 3B subgroups of patients (online supplemental table 1). Patients with CKD stage 3B had a slightly longer mean duration of T2DM (16.7 \pm 9.3 years) and less frequent use of metformin $(45.1 \%)$ but more frequent use of insulin $(68.5 \%)$ compared with patients with CKD stage 3A (mean duration of T2DM: $15.3 \pm 8.8$ years; metformin use: $68.9 \%$; insulin use: $54.7 \%$ ). In line with a more advanced stage of kidney function impairment, patients with CKD stage 3B also had a lower mean eGFR $\left(38.8 \pm 4.0 \mathrm{~mL} / \mathrm{min} / 1.73 \mathrm{~m}^{2}\right)$ and a higher prevalence of macro-albuminuria $(21.9 \%)$ at baseline compared with patients with CKD stage 3A (mean eGFR: $53.0 \pm 4.3 \mathrm{~mL} /$ $\mathrm{min} / 1.73 \mathrm{~m}^{2}$; macro-albuminuria: $13.5 \%$ ).

\section{HbA1c efficacy outcomes}

In the subgroup of VERTIS CV patients with CKD stage 3, significant reductions in HbAlc over 18 weeks were observed with ertugliflozin $5 \mathrm{mg}$ and $15 \mathrm{mg}$ versus placebo (figure 1A). The placebo-adjusted LS-mean changes from baseline $(95 \% \mathrm{CI})$ to week 18 were $-0.27 \%(-0.37 \%$ to $-0.17 \%)$ with ertugliflozin $5 \mathrm{mg}$ and $-0.28 \%$ ( $-0.38 \%$ to $-0.17 \%)$ with ertugliflozin $15 \mathrm{mg}$ (both $\mathrm{p}<0.001$ ). Long-term change in HbA1c over time in the CKD stage 3 subgroup of patients is shown in figure 1B. Greater reductions in HbAlc with ertugliflozin, compared with placebo, were observed over 4 years.

Significant reductions in HbAlc were observed with both ertugliflozin doses versus placebo in the subgroup of patients with CKD stage 3A (figure 1A). The adjusted mean changes from baseline to week 18 were $-0.27 \%$ $(-0.38 \%$ to $-0.15 \%)$ with ertugliflozin $5 \mathrm{mg}$ and $-0.31 \%$ 
Table 1 Baseline demographics and clinical characteristics of VERTIS CV patients with CKD stage $3^{*}$

\begin{tabular}{|c|c|c|c|}
\hline Characteristic $†$ & $\begin{array}{l}\text { Placebo } \\
(\mathrm{N}=598)\end{array}$ & $\begin{array}{l}\text { Ertugliflozin } 5 \mathrm{mg} \\
(\mathrm{N}=618)\end{array}$ & $\begin{array}{l}\text { Ertugliflozin } 15 \mathrm{mg} \\
(\mathrm{N}=560)\end{array}$ \\
\hline Age-years & $68.0(7.5)$ & $68.3(7.7)$ & $68.2(7.5)$ \\
\hline Male-n (\%) & $395(66.1)$ & $396(64.1)$ & $351(62.7)$ \\
\hline \multicolumn{4}{|l|}{ Race-n (\%)‡ } \\
\hline White & $514(86.0)$ & $523(84.6)$ & $493(88.0)$ \\
\hline Black & $17(2.8)$ & $21(3.4)$ & $9(1.6)$ \\
\hline Asian & $43(7.2)$ & $55(8.9)$ & $35(6.3)$ \\
\hline Other & $24(4.0)$ & $19(3.1)$ & $23(4.1)$ \\
\hline \multicolumn{4}{|l|}{ Region-n (\%) } \\
\hline North America & $191(31.9)$ & $189(30.6)$ & $169(30.2)$ \\
\hline South America & $46(7.7)$ & $42(6.8)$ & $49(8.8)$ \\
\hline Europe & $271(45.3)$ & $290(46.9)$ & $262(46.8)$ \\
\hline Asia & $46(7.7)$ & $49(7.9)$ & $32(5.7)$ \\
\hline South Africa & $28(4.7)$ & $37(6.0)$ & $30(5.4)$ \\
\hline Australia/New Zealand & $16(2.7)$ & $11(1.8)$ & $18(3.2)$ \\
\hline Body mass index $-\mathrm{kg} / \mathrm{m}^{2}$ & $32.6(5.6)$ & $32.3(5.3)$ & $32.4(5.7)$ \\
\hline Duration of type 2 diabetes - years & $16.4(9.3)$ & $15.3(8.8)$ & $15.2(8.7)$ \\
\hline Glycated hemoglobin-\% & $8.2(0.9)$ & $8.2(0.9)$ & $8.2(0.9)$ \\
\hline eGFR-mL/min $/ 1.73 \mathrm{~m}^{2} \S$ & $49.0(7.5)$ & $49.6(7.4)$ & $49.4(7.7)$ \\
\hline \multicolumn{4}{|l|}{ Albuminuria-n (\%) } \\
\hline Normal-albuminuria $(<30 \mathrm{mg} / \mathrm{g})$ & $285(47.7)$ & $302(48.9)$ & $268(47.9)$ \\
\hline Micro-albuminuria $(\geq 30-\leq 300 \mathrm{mg} / \mathrm{g})$ & $207(34.6)$ & $209(33.8)$ & $178(31.8)$ \\
\hline Macro-albuminuria (>300 mg/g) & $92(15.4)$ & $91(14.7)$ & $95(17.0)$ \\
\hline Unknown & $14(2.3)$ & $16(2.6)$ & $19(3.4)$ \\
\hline Systolic blood pressure $-\mathrm{mm} \mathrm{Hg}$ & $132.7(15.2)$ & $134.7(14.8)$ & $133.6(14.5)$ \\
\hline Hypertension $-n(\%)$ & $571(95.5)$ & $589(95.3)$ & $524(93.6)$ \\
\hline Dyslipidemia-n (\%) & $489(81.8)$ & $496(80.3)$ & $445(79.5)$ \\
\hline Coronary artery disease $-\mathrm{n}(\%)$ & $480(80.3)$ & $491(79.4)$ & $436(77.9)$ \\
\hline Cerebrovascular disease $-\mathrm{n}(\%)$ & $138(23.1)$ & $153(24.8)$ & $147(26.3)$ \\
\hline Peripheral arterial disease $-\mathrm{n}(\%)$ & $123(20.6)$ & $113(18.3)$ & $111(19.8)$ \\
\hline \multicolumn{4}{|l|}{ Antihyperglycemic medications $-\mathrm{n}(\%)$} \\
\hline None & $3(0.5)$ & $5(0.8)$ & $0(0.0)$ \\
\hline Metformin & $382(63.9)$ & $385(62.3)$ & $348(62.1)$ \\
\hline Insulin & $357(59.7)$ & $370(59.9)$ & $308(55.0)$ \\
\hline Sulfonylurea & $227(38.0)$ & $231(37.4)$ & $219(39.1)$ \\
\hline Dipeptidyl peptidase-4 inhibitor & $72(12.0)$ & 74 (12.0) & 70 (12.5) \\
\hline Glucagon-like peptide- 1 receptor agonist & $25(4.2)$ & $25(4.0)$ & $19(3.4)$ \\
\hline
\end{tabular}

${ }^{*} \mathrm{CKD}$ stage was based on eGFR calculated using the MDRD equation.

†Values are mean \pm SD unless otherwise stated.

‡Race was reported by the participant.

§eGFR was calculated using the MDRD equation.

CKD, chronic kidney disease; CKD stage 3, eGFR 30-<60 mL/min/1.73 m²; eGFR, estimated glomerular filtration rate; MDRD, Modification of Diet in Renal Disease.

$(-0.43 \%$ to $-0.19 \%)$ with ertugliflozin $15 \mathrm{mg}$ (both $\mathrm{p}<0.001)$. In the subgroup of patients with CKD stage 3B, a significant placebo-adjusted reduction in HbAlc from baseline to week 18 was observed with the $5 \mathrm{mg}$ dose of ertugliflozin $(-0.28 \% \quad(-0.47 \%$ to $-0.08 \%)$; $\mathrm{p}=0.006$ ) (figure 1A) but the observed reduction in HbAlc was not statistically significant for the $15 \mathrm{mg}$ dose $(-0.19 \%(-0.39 \%$ to $0.01 \%) ; \mathrm{p}=0.064)$ (figure $1 \mathrm{~A})$. 

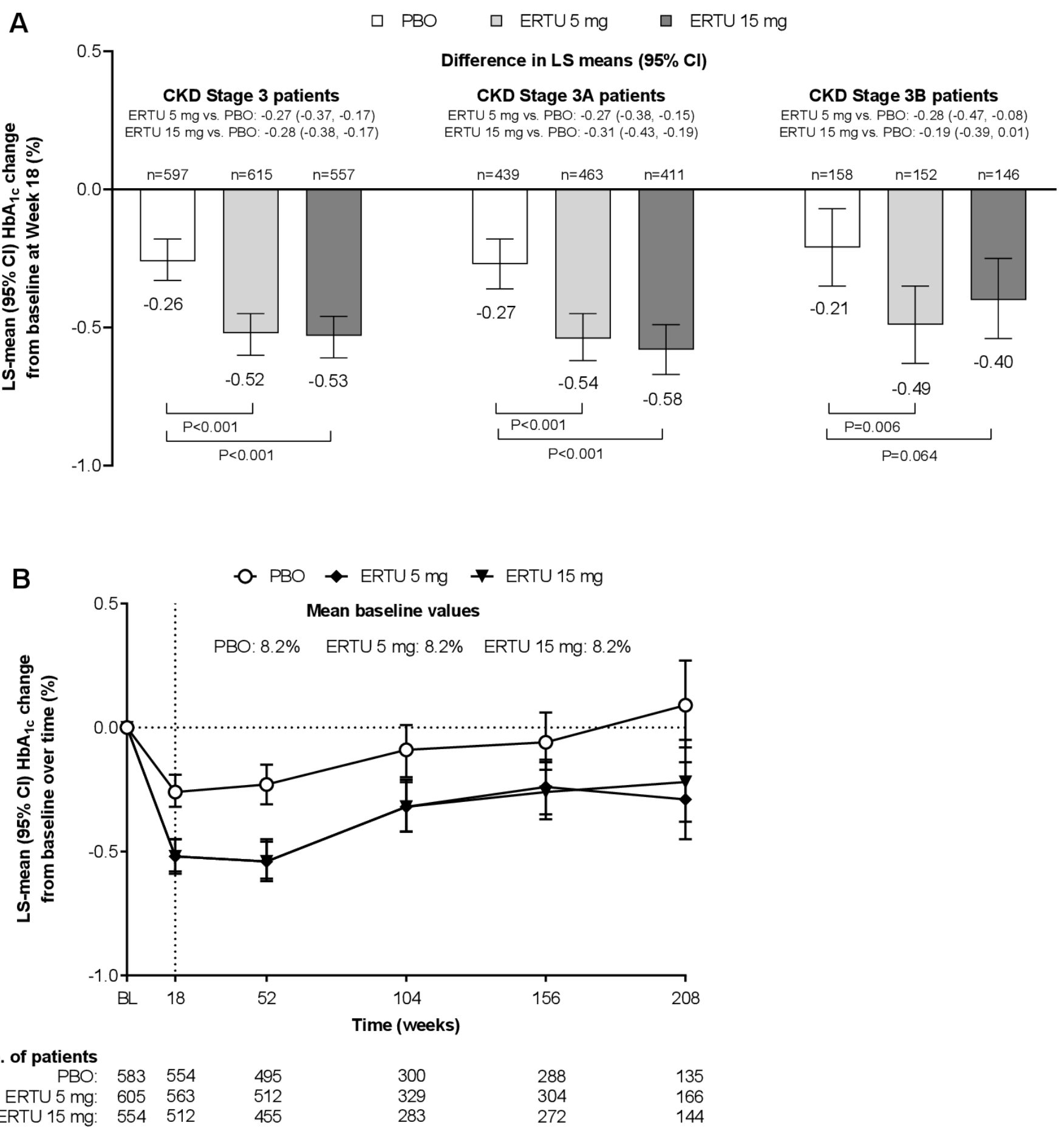

Figure 1 (A) HbA1c efficacy at week 18 in VERTIS CV patients with CKD stage 3, CKD stage 3A, and CKD stage 3B.

Calculated using a cLDA model with fixed effects for treatment, time (categorical), baseline (BL) eGFR (continuous), and the interaction of time-by-treatment. $P$ values are nominal; $n$ is the number of patients in the cLDA FAS (ie, randomized patients who took at least one dose of study medication and had at least one assessment at or after BL) with non-missing assessments at BL. (B) HbA1c efficacy over time in VERTIS CV patients with CKD stage 3. Calculated using an ANCOVA model adjusted for baseline $\mathrm{HbA1c}$, treatment, time, subgroup, treatment-by-subgroup interaction, and treatment-by-subgroup-by-time interaction; $n$ is the number of patients in the ANCOVA FAS (ie, randomized patients who took at least one dose of study medication and had a BL and at least one post-BL assessment) with non-missing assessments at the specific time point. CKD stage was based on eGFR calculated using the MDRD equation. ANCOVA, analysis of covariance; CKD, chronic kidney disease; CKD stage 3, eGFR 30-<60 mL/min $/ 1.73 \mathrm{~m}^{2}$; CKD stage 3A, eGFR 45-<60 mL/min $/ 1.73 \mathrm{~m}^{2}$; CKD stage 3B, eGFR $30-<45 \mathrm{~mL} / \mathrm{min} / 1.73 \mathrm{~m}^{2}$; CLDA, constrained longitudinal data analysis; eGFR, estimated glomerular filtration rate; ERTU, ertugliflozin; FAS, full analysis set; HbA1c, glycated hemoglobin; LS, least squares; MDRD, Modification of Diet in Renal Disease; PBO, placebo. 
Table 2 Other efficacy outcomes at week 18 in VERTIS CV patients with CKD stage $3^{*}$

\begin{tabular}{|c|c|c|c|}
\hline Efficacy outcome & Placebo & Ertugliflozin $5 \mathrm{mg}$ & Ertugliflozin $15 \mathrm{mg}$ \\
\hline \multicolumn{4}{|l|}{$\mathrm{HbA} 1 \mathrm{c}<7.0 \%$} \\
\hline $\mathrm{N} \dagger$ & 597 & 617 & 559 \\
\hline Number (\%) with $\mathrm{HbA} 1 \mathrm{c}<7.0 \%$ & $77(12.9)$ & $128(20.7)$ & $95(17.0)$ \\
\hline Adjusted OR vs placebo $(95 \% \mathrm{Cl}) \ddagger$ & - & 1.79 (1.28 to 2.50$)$ & 1.46 (1.04 to 2.05$)$ \\
\hline$P$ value vs placebo $\ddagger$ & - & $<0.001$ & 0.03 \\
\hline \multicolumn{4}{|l|}{ FPG } \\
\hline $\mathrm{n} / \mathrm{N} \dagger$ & $594 / 597$ & $616 / 617$ & $557 / 559$ \\
\hline Baseline mean (SD) $-\mathrm{mg} / \mathrm{dL}$ & $172.3(52.95)$ & $175.2(55.31)$ & $173.1(58.52)$ \\
\hline LS-mean change from baseline $(95 \% \mathrm{CI}) \S$ & $-22.78(-26.55$ to -19.02$)$ & $-30.63(-34.23$ to -27.03$)$ & $-31.99(-35.69$ to -28.29$)$ \\
\hline Difference in LS-mean vs placebo $(95 \% \mathrm{CI}) \S$ & - & $-7.85(-12.23$ to -3.47$)$ & $-9.21(-13.67$ to -4.74$)$ \\
\hline$P$ value vs placebo & - & $<0.001$ & $<0.001$ \\
\hline \multicolumn{4}{|l|}{ Body weight } \\
\hline $\mathrm{n} / \mathrm{N} \dagger$ & $597 / 597$ & $617 / 617$ & $560 / 560$ \\
\hline Baseline mean (SD) - kg & $92.5(19.04)$ & $91.6(17.85)$ & $91.6(19.35)$ \\
\hline LS-mean change from baseline $(95 \% \mathrm{Cl}) \S$ & $-0.41(-0.65$ to -0.17$)$ & $-1.77(-2.01$ to -1.54$)$ & $-1.93(-2.18$ to -1.69$)$ \\
\hline Difference in LS-mean vs placebo $(95 \% \mathrm{CI}) \S$ & - & $-1.37(-1.70$ to -1.04$)$ & $-1.53(-1.86$ to -1.19$)$ \\
\hline$P$ value vs placebo & - & $<0.001$ & $<0.001$ \\
\hline \multicolumn{4}{|l|}{ SBP } \\
\hline $\mathrm{n} / \mathrm{N} \dagger$ & $597 / 597$ & $615 / 617$ & $560 / 560$ \\
\hline Baseline mean (SD) $-\mathrm{mm} \mathrm{Hg}$ & $132.7(15.18)$ & $134.7(14.85)$ & $133.6(14.47)$ \\
\hline LS-mean change from baseline $(95 \% \mathrm{CI}) \S$ & $0.90(-0.30$ to 2.09$)$ & $-1.99(-3.15$ to -0.83$)$ & $-2.29(-3.50$ to -1.08$)$ \\
\hline Difference in LS-mean vs placebo $(95 \% \mathrm{CI}) \S$ & - & $-2.89(-4.48$ to -1.30$)$ & $-3.19(-4.81$ to -1.56$)$ \\
\hline$P$ value vs placebol & - & $<0.001$ & $<0.001$ \\
\hline
\end{tabular}

${ }^{*}$ CKD stage was based on eGFR calculated using the MDRD equation.

$\mathrm{tn}$ is the number of patients with non-missing assessments at baseline; $\mathrm{N}$ is the number of patients in the cLDA FAS (ie, randomized patients who took at least one dose of study medication and had at least one assessment at or after baseline).

$\ddagger$ Adjusted OR based on a logistic regression model fitted with fixed effects for treatment, and covariates for baseline HbA1c and baseline eGFR (continuous). Missing data were imputed using the cLDA model fitted with fixed effects as in the primary analysis.

$\S$ Calculated using a cLDA model with fixed effects for treatment, time, baseline eGFR (continuous), and the interaction of time (categorical) by treatment.

ףNominal $p$ values.

CKD, chronic kidney disease; CKD stage 3, eGFR 30-<60 mL/min/1.73 m²; cLDA, constrained longitudinal data analysis; eGFR, estimated glomerular filtration rate; FAS, full analysis set; FPG, fasting plasma glucose; HbA1c, glycated hemoglobin; LS, least squares; MDRD, Modification of Diet in Renal Disease; SBP, systolic blood pressure.

In the subgroup of patients with CKD stage 3, the proportion of patients who had HbAlc $<7.0 \%$ at week 18 was greater in the ertugliflozin $5 \mathrm{mg}$ and $15 \mathrm{mg}$ groups compared with placebo (table 2).

\section{Other efficacy outcomes}

In patients with CKD stage 3 , both the 5 and $15 \mathrm{mg}$ doses of ertugliflozin, compared with placebo, were associated with significant reductions in FPG, body weight, and SBP over 18 weeks (table 2). Significant reductions in FPG from baseline to week 18 were observed with ertugliflozin versus placebo in patients with CKD stage $3 \mathrm{~A}$; however, in the subgroup of patients with CKD stage 3B, reductions in FPG were not statistically significant (online supplemental table 2). Both the 5 and $15 \mathrm{mg}$ doses of ertugliflozin significantly reduced body weight in the CKD stage $3 \mathrm{~A}$ and stage $3 \mathrm{~B}$ subgroups of patients (online supplemental table 2). Compared with placebo, ertugliflozin significantly reduced SBP over 18 weeks in the subgroup of patients with CKD stage 3A; however, in the subgroup of patients with CKD stage 3B, reductions in SBP were not statistically significant (online supplemental table 2).

\section{Safety outcomes}

In the subgroup of patients with CKD stage 3, the incidence of AEs, SAEs, AEs leading to study drug discontinuation, and deaths did not differ across the ertugliflozin and placebo treatment groups (table 3). An analysis of prespecified AEs of interest revealed a similar incidence of UTIs with ertugliflozin and placebo in patients with CKD stage 3 (table 3). An increased incidence of GMIs was observed with ertugliflozin treatment compared with placebo in both women and men (table 3 ). The proportion of patients experiencing events of symptomatic hypoglycemia or hypovolemia was well balanced across placebo and ertugliflozin treatment groups in patients with CKD stage 3 (table 3 ). 
Table 3 Adverse events in VERTIS CV patients with CKD stage $3^{*}$

\begin{tabular}{|c|c|c|c|}
\hline Adverse event $\dagger$ & $\begin{array}{l}\text { Placebo } \\
(\mathrm{N}=597) \ddagger\end{array}$ & $\begin{array}{l}\text { Ertugliflozin } 5 \mathrm{mg} \\
(\mathrm{N}=617) \ddagger\end{array}$ & $\begin{array}{l}\text { Ertugliflozin } 15 \mathrm{mg} \\
(\mathrm{N}=560) \ddagger\end{array}$ \\
\hline Any $A E-n(\%)$ & $535(89.6)$ & $561(90.9)$ & $501(89.5)$ \\
\hline Serious AEs-n (\%) & $250(41.9)$ & $252(40.8)$ & $222(39.6)$ \\
\hline AEs leading to discontinuation of study drug $-\mathrm{n}(\%)$ & $51(8.5)$ & $66(10.7)$ & $54(9.6)$ \\
\hline Deaths -n (\%) & $28(4.7)$ & $34(5.5)$ & $30(5.4)$ \\
\hline \multicolumn{4}{|l|}{ Prespecified AEs of interest $-n(\%)$} \\
\hline Urinary tract infection & 77 (12.9) & $85(13.8)$ & 79 (14.1) \\
\hline Genital mycotic infection-women§ & $8(3.9)$ & $9(4.1)$ & $17(8.1)$ \\
\hline Genital mycotic infection-men $\mathbb{q}$ & $1(0.3)$ & $12(3.0)$ & $13(3.7)$ \\
\hline Symptomatic hypoglycemia ${ }^{\star \star}$ & $224(37.5)$ & $232(37.6)$ & $193(34.5)$ \\
\hline Hypovolemia & $39(6.5)$ & $44(7.1)$ & $33(5.9)$ \\
\hline \multicolumn{4}{|l|}{ Kidney-related AEs-n (\%) } \\
\hline Any kidney-related $\mathrm{AE}$ & $61(10.2)$ & $60(9.7)$ & $56(10.0)$ \\
\hline Acute kidney injury & $32(5.4)$ & $23(3.7)$ & $30(5.4)$ \\
\hline Kidney failure & $17(2.8)$ & $14(2.3)$ & $11(2.0)$ \\
\hline Kidney impairment & $18(3.0)$ & $28(4.5)$ & $17(3.0)$ \\
\hline \multicolumn{4}{|l|}{ Adjudicated kidney events-n (\%) } \\
\hline Any adjudicated kidney event & 24 & 16 & 21 \\
\hline \multicolumn{4}{|l|}{ Causality } \\
\hline Very likely & $0(0.0)$ & $0(0.0)$ & $0(0.0)$ \\
\hline Probable & $0(0.0)$ & $0(0.0)$ & $0(0.0)$ \\
\hline Possible & $4(16.7)$ & $0(0.0)$ & $3(14.3)$ \\
\hline Doubtful & $7(29.2)$ & $10(62.5)$ & $8(38.1)$ \\
\hline Not related & $13(54.2)$ & $6(37.5)$ & $10(47.6)$ \\
\hline
\end{tabular}

${ }^{*}$ CKD stage was based on eGFR calculated using the MDRD equation.

†All events occurring within 14 days of final dose of treatment and including events after initiation of glycemic rescue medication, except for those related to hypoglycemia.

$\ddagger \mathrm{N}$ is the number of patients in the safety analysis population (ie, patients who took at least one dose of study medication).

$\S$ Number of women with CKD stage 3: placebo, $n=203$; ertugliflozin $5 \mathrm{mg}, \mathrm{n}=222$; ertugliflozin $15 \mathrm{mg}, \mathrm{n}=209$.

INumber of men with CKD stage 3: placebo, $n=394$; ertugliflozin $5 \mathrm{mg}, \mathrm{n}=395$; ertugliflozin $15 \mathrm{mg}, \mathrm{n}=351$.

**Symptomatic hypoglycemia was defined as an event with clinical symptoms reported by the investigator as hypoglycemia (biochemical documentation not required).

$\mathrm{AE}$, adverse event; CKD, chronic kidney disease; CKD stage 3, eGFR 30-<60 mL/min/1.73 $\mathrm{m}^{2}$; eGFR, estimated glomerular filtration rate; MDRD, Modification of Diet in Renal Disease.

The incidence of kidney-related AEs, including the incidence of acute kidney injury, was similar with placebo and ertugliflozin treatment in patients with CKD stage 3 (table 3). The occurrence of kidney-related AEs meeting prespecified criteria for adjudication was also similar across treatment groups in patients with CKD stage 3 . The majority of adjudicated kidney events were assessed as having 'Doubtful' or 'Not related' causality to the study medication (table 3). No events were assessed as 'Very Likely' or 'Probable' and few events were adjudicated as 'Possible' with no evidence of increased frequency associated with ertugliflozin treatment.

The general pattern of AEs observed between treatment groups in the subgroups of patients with CKD stage $3 \mathrm{~A}$ and stage $3 \mathrm{~B}$ (online supplemental table 3 ) was consistent with that seen in the overall group of patients with CKD stage 3 (table 3), including the incidence of AEs, SAEs, AEs leading to study drug discontinuation, deaths, prespecified AEs of interest, and kidney-related AEs. Although the incidence of kidney-related AEs was balanced across placebo and ertugliflozin treatment groups in patients with CKD stage $3 \mathrm{~A}$ and CKD stage $3 \mathrm{~B}$, kidney-related AEs were more frequently reported in patients with CKD stage 3B across all treatment groups (online supplemental table 3), consistent with having a more advanced impairment in kidney function.

Compared with placebo, initiation of ertugliflozin treatment was associated with an early dip in eGFR that was transient and followed by eGFR levels that were maintained above or near baseline (figure 2A). The pattern of change from baseline in eGFR over time observed in the subgroups of patients with CKD stage 3A (figure 2B) and 
A

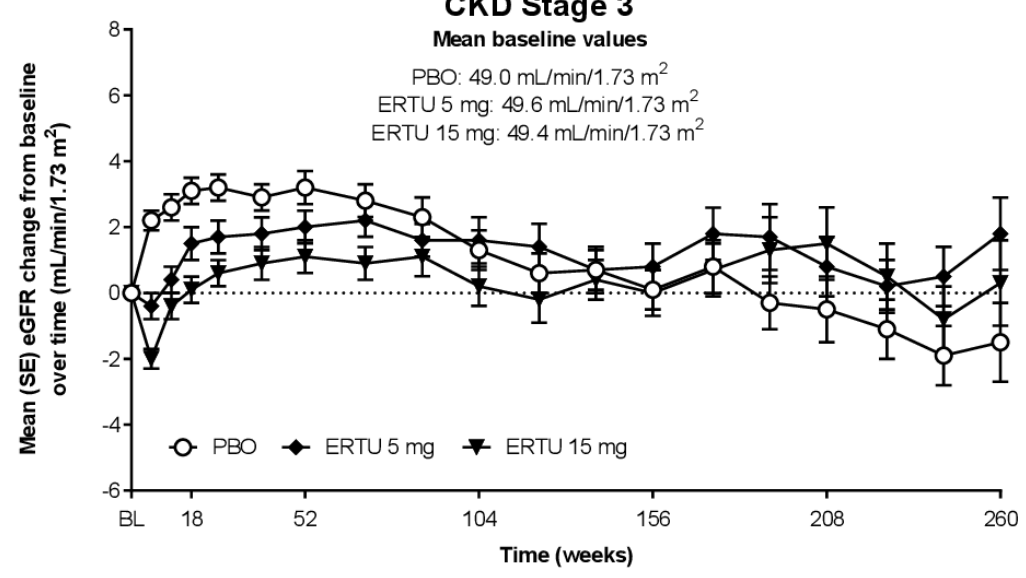

No. of patients

$\begin{array}{rllllllr}\text { PBO: } & 597 & 545 & 486 & 298 & 283 & 134 & 86 \\ \text { ERTU 5 mg: } & 617 & 557 & 508 & 333 & 301 & 167 & 106 \\ \text { ERTU 15 mg: } & 560 & 504 & 449 & 282 & 272 & 142 & 86\end{array}$

B

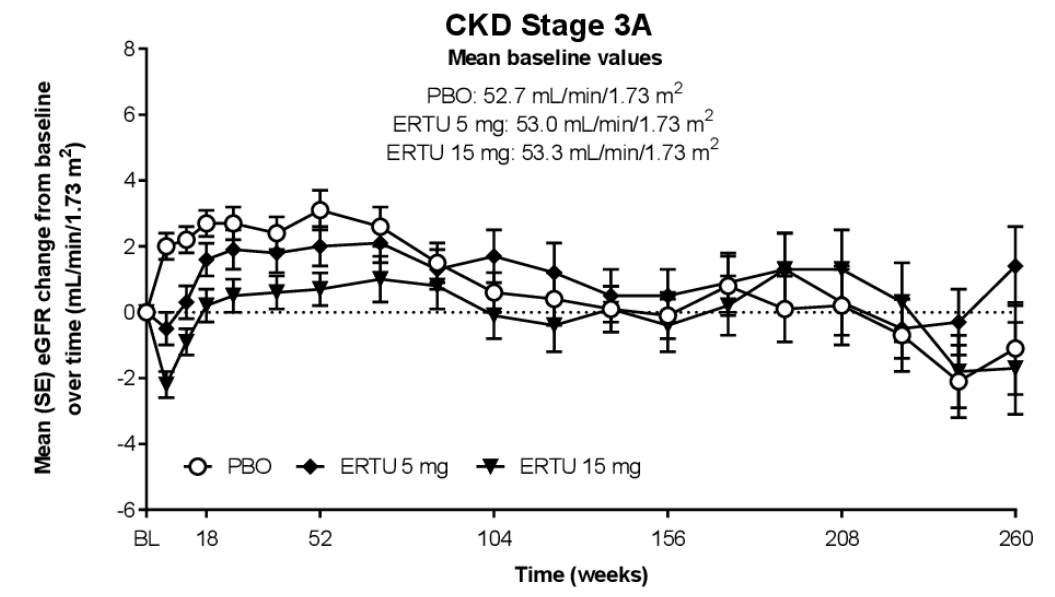

Figure 2 Mean $( \pm S E)$ change from baseline (BL) in eGFR over time in VERTIS CV patients with (A) CKD stage 3, (B) CKD stage $3 A$, and (C) CKD stage 3B. CKD stage was based on eGFR calculated using the MDRD equation. $n$ is the number of patients with non-missing assessments at both $\mathrm{BL}$ and the specific time point. CKD, chronic kidney disease; CKD stage 3, eGFR $30-<60 \mathrm{~mL} / \mathrm{min} / 1.73 \mathrm{~m}^{2} ;$ CKD stage 3A, eGFR $45-<60 \mathrm{~mL} / \mathrm{min} / 1.73 \mathrm{~m}^{2} ;$ CKD stage 3B, eGFR $30-<45 \mathrm{~mL} / \mathrm{min} / 1.73 \mathrm{~m}^{2} ;$ eGFR, estimated glomerular filtration rate; ERTU, ertugliflozin; MDRD, Modification of Diet in Renal Disease; PBO, placebo. 
CKD stage 3B (figure 2C) was also generally similar to that seen in the overall group of patients with CKD stage 3 (figure 2A).

\section{DISCUSSION}

The inclusion criteria for the VERTIS CV study allowed the entry of patients with an eGFR $\geq 30 \mathrm{~mL} / \mathrm{min} / 1.73 \mathrm{~m}^{2}$, which resulted in the randomization of $21.5 \%$ (1776 of 8246) of patients with CKD stage 3 in the trial. Among those with CKD stage 3, 74\% had CKD stage 3A and $\sim 26 \%$ had CKD stage 3B. The inclusion of these patients in the VERTIS CV trial allowed a comprehensive assessment of ertugliflozin glycemic efficacy and safety in patients with T2DM, ASCVD, and CKD stage 3.

The use of data from the initial 18 weeks of VERTIS $\mathrm{CV}$ was designed to allow a comparison with placebo as doses of background antihyperglycemic medications were held stable during this period; after week 18 , investigators were encouraged to optimize glycemic therapy to achieve individualized treatment goals. The present analyses demonstrated that both doses of ertugliflozin $(5 \mathrm{mg}$ and $15 \mathrm{mg}$ ) resulted in statistically significant placeboadjusted reductions in $\mathrm{HbA1c}$ from baseline at week 18 in the overall CKD stage 3 subgroup and in the CKD stage 3A subgroup. In the CKD stage 3B subgroup, the ertugliflozin $15 \mathrm{mg}$ dose did not achieve statistical significance, compared with placebo, and may be due to the relatively small patient numbers in this subgroup. Another contributing factor to the apparent absence of dose-response in the CKD stage 3B subgroup could be the greater degree of diminished kidney function in this subgroup in the context of the dose-response curve of ertugliflozin (model-predicted HbAlc and 24-hour urinary glucose excretion responses for ertugliflozin $5 \mathrm{mg}$ and $15 \mathrm{mg}$ doses are $>80 \%$ and $>90 \%$, respectively, of the maximum model-predicted response).$^{17}$

The observed reductions from baseline at week 18 in FPG roughly paralleled those observed for HbAlc. The reduction in $\mathrm{HbA1c}$ in the CKD stage $3 \mathrm{~B}$ group, despite the lack of a significant decrease in FPG levels, likely resulted from suppression of postprandial plasma glucose, a stronger contributor to HbAlc than FPG. Although the longer term assessment of HbAlc efficacy for CKD stage 3 overall is confounded by changes in background medication that were encouraged to meet individualized glycemic goals after 18 weeks, the data indicate that ertugliflozin treatment, compared with placebo, was associated with consistently better glycemic control over 4 years. In the VERTIS CV trial protocol, ${ }^{14}$ participating clinics were encouraged to optimize glycemic control after 18 weeks, using agents from all antihyperglycemic classes except SGLT2 inhibitors. Thus, the continued separation of the HbAlc curves between the placebo group and the ertugliflozin groups most likely reflects practice patterns by the study investigators. ${ }^{15}$ However, the durable efficacy observed in the ertugliflozin-treated groups could also be due to the weight loss (which improves glycemic control) and/or preservation of kidney function (which supports the mechanism of action of SGLT2 inhibitors) that was observed in these patients.

Although glycemic parameters following ertugliflozin treatment in the CKD stage 3 and stage $3 \mathrm{~A}$ categories were statistically significantly different from placebo, as anticipated by the SGLT2 inhibitor mechanism of action within the kidney, the reductions in HbAlc in the CKD stage 3 categories were attenuated compared with the HbA1c reductions with ertugliflozin $5 \mathrm{mg}$ and $15 \mathrm{mg}$ observed in a pooled analysis of placebo-controlled VERTIS phase 3 glycemic efficacy studies that did not include VERTIS CV. ${ }^{18}$ In those studies, in which exclusion criteria required randomized patients to have an eGFR $\geq 55 \mathrm{~mL} / \mathrm{min} / 1.73 \mathrm{~m}^{2}$ (average eGFR was $\sim 89 \mathrm{~mL}$ / $\min / 1.73 \mathrm{~m}^{2}$ ), the changes from baseline in HbAlc at week 26 were $-0.8 \%$ and $-0.9 \%$ for ertugliflozin $5 \mathrm{mg}$ and $15 \mathrm{mg}$, respectively. ${ }^{18}$

The magnitude of the HbAlc reductions observed in this analysis of VERTIS CV CKD stage 3, 3A, and 3B subgroups is consistent with that observed for other SGLT2 inhibitors in this patient population. In a subgroup analysis of the EMPA-REG OUTCOME trial, ${ }^{19}$ the placebo-adjusted change from baseline at week 12 in HbA1c in patients with CKD stage 3 treated with empagliflozin $10 \mathrm{mg}$ and $25 \mathrm{mg}$ was $-0.36 \%$ for each dose group. The placebo-adjusted changes from baseline at week 12 in HbAlc in patients with CKD stage $3 \mathrm{~A}$ and stage $3 \mathrm{~B}$ (pooled $10 \mathrm{mg}$ and $25 \mathrm{mg}$ doses) were $-0.43 \%$ and $-0.21 \%$, respectively. ${ }^{19}$ Similarly, in a subgroup analysis of CANVAS trial data, ${ }^{20}$ the placebo-adjusted change from baseline at week 18 in $\mathrm{HbA1c}$ in patients with CKD stage 3 treated with canagliflozin (pooled $100 \mathrm{mg}$ and $300 \mathrm{mg}$ doses) was $-0.42 \%$, and in the subgroups of patients with CKD stage $3 \mathrm{~A}$ and stage $3 \mathrm{~B}$ was $-0.45 \%$ and $-0.35 \%$, respectively. In the DERIVE study, the placebo-adjusted change from baseline at week 24 in HbAlc in patients with CKD stage 3A treated with dapagliflozin $10 \mathrm{mg}$ was $-0.34 \% .^{21}$ The HbAlc reductions observed in the present analysis are also consistent with the previous ertugliflozin study in patients with moderately impaired kidney function (VERTIS RENAL). ${ }^{22}$ In contrast to the attenuated glycemic efficacy of SGLT2 inhibitors observed in patients with CKD stage 3, previous studies have reported a more potent HbA1c-lowering effect of SGLT2 inhibitors in patients with preserved kidney function, with placebosubtracted $\mathrm{HbAlc}$ reductions from baseline of $-0.6 \%$ to $-0.8 \%$ in monotherapy studies. ${ }^{23} 24$

Although HbA1c efficacy was attenuated in this patient population, reductions in body weight and SBP with ertugliflozin in patients with CKD stage 3 were similar to those observed in patients with preserved kidney function in a pooled analysis of placebocontrolled VERTIS phase 3 studies. ${ }^{18}$ The similar body weight and SBP reductions in patients with varying degrees of kidney function suggest that the effects of ertugliflozin on body weight and SBP are independent from glucosuria. 
In the present analyses of VERTIS CV patients with CKD stage 3, the safety of ertugliflozin was consistent with that observed in the overall population followed over the duration of the VERTIS CV study and consistent with the known safety profile of ertugliflozin from the VERTIS phase 3 development program. ${ }^{15}{ }^{25}$ Incidences of summary AE measures, kidney-related AEs, and adjudicated kidney events were similar in the ertugliflozin and placebo groups within the CKD stage 3, stage 3A, and stage 3B subgroups of patients. Symptomatic hypoglycemia was also similar across treatment groups within each of the CKD categories. The incidence of GMIs was higher in both men and women in the ertugliflozin groups within each CKD category compared with placebo.

The observation that after the initial dip in eGFR with ertugliflozin treatment, eGFR returned to baseline and remained near or above baseline to the end of the study in patients with CKD stage 3, as opposed to remaining below baseline as was observed in the overall VERTIS $\mathrm{CV}$ population, ${ }^{15}$ is consistent with the results from $\mathrm{CV}$ outcomes trials of other SGLT2 inhibitors. ${ }^{202627}$ As is the case for the long-term pattern of changes in eGFR observed in the overall VERTIS CV population, the present results also suggest an effect of ertugliflozin on the preservation of kidney function in patients with baseline eGFR levels between 30 and $60 \mathrm{~mL} / \mathrm{min} / 1.73 \mathrm{~m}^{2}$.

The goal of the VERTIS CV trial was to attempt to achieve glycemic equipoise between the placebo and ertugliflozin groups. Although it is unlikely the modest reductions in HbAlc observed in the CKD stage 3 subgroup contributed significantly to cardiorenal outcomes in these patients, the Diabetes Control and Complications Trial and other landmark studies have demonstrated a linear relationship between HbAlc reduction and decreased risk of microvascular complications (reviewed in reference 28). From that perspective, reductions in $\mathrm{HbAlc}$ of the magnitude observed in this analysis would be clinically meaningful. Moreover, the attenuated glycemic efficacy observed with SGLT2 inhibitors in patients with CKD stage 3 should be viewed within the broader context of other effects of SGLT2 inhibitors in this patient population who are at high risk of further deterioration of kidney function over time and of HHF. Preservation of kidney function and reduction in the risk of $\mathrm{HHF}$ in patients with CKD stage 3 have been demonstrated by other members of the SGLT2 inhibitor class. $^{27}$ 29-31 In VERTIS CV, prespecified secondary analyses have demonstrated that treatment with ertugliflozin, compared with placebo, slowed the deterioration of kidneyfiltering capacity (eGFR), reduced albuminuria (UACR), and reduced progression and increased regression of albuminuria across a range of baseline kidney function subgroups defined by baseline eGFR (including CKD stage 3), albuminuria, and Kidney Disease: Improving Global Outcomes risk categories. ${ }^{32}$ In VERTIS CV, the assessment of HHF risk was a prespecified secondary objective. The assessment showed that ertugliflozin treatment, compared with placebo, was associated with a $30 \%$ reduction in the risk of HHF. ${ }^{153}$ In addition, the effect of ertugliflozin on reducing the risk of HHF was greater (higher relative risk reductions and absolute risk reductions) in the subgroup of VERTIS CV patients with CKD stage $3 .^{33}$

There are several potential limitations to the present analysis. The analysis was a subgroup analysis rather than a dedicated study in patients with CKD stage 3 . The duration of the placebo-controlled period was limited to 18 weeks. However, maximal HbAlc efficacy is typically observed after initiation of SGLT2 inhibitor therapy by week 12, and the observed response should be useful to assist clinicians in setting expectations for glycemic control for these patients. This substudy was conducted exclusively in patients with T2DM and ASCVD; nonetheless, the findings are likely to be broadly applicable to patients with T2DM without prevalent ASCVD. Finally, the study population was predominantly white $(\sim 86 \%)$, which limits the generalizability of our findings to individuals from under-represented populations.

In conclusion, the present analyses of VERTIS CV patients with CKD stage 3 demonstrated reductions in glycemic parameters (HbA1c and FPG), body weight, and SBP similar to those observed for other members of the SGLT2 inhibitor class. These results, and results from the main VERTIS CV study, suggest that ertugliflozin preserves kidney function and reduces the risk of HHF, in addition to providing a modest degree of glycemic control in patients with CKD stage 3. The totality of these effects indicates a positive benefit/risk profile of ertugliflozin in patients with T2DM, ASCVD, and CKD stage 3. The present findings add to the body of evidence on the effects of SGLT2 inhibitors in patients with T2DM and CKD stage 3, and provide further support for recent society guidelines for the use of this drug class in this patient population. ${ }^{9-11}$

\section{Author affiliations}

${ }^{1}$ Department of Medicine, University of Tennessee Health Science Center, Memphis, Tennessee, USA

${ }^{2}$ AdventHealth Translational Research Institute, Orlando, Florida, USA

${ }^{3}$ University Health Network, University of Toronto, Toronto, Ontario, Canada

${ }^{4}$ Division of Cardiology, Department of Internal Medicine, University of Texas Southwestern Medical Center and Parkland Health \& Hospital System, Dallas,

Texas, USA

${ }^{5}$ Unit of Cardiology, Karolinska Institute and Karolinska University Hospital, Stockholm, Sweden

${ }^{6}$ Department of Biostatistics, Rutgers School of Public Health and Rutgers Cancer Institute of New Jersey, Piscataway, New Jersey, USA

${ }^{7}$ Merck \& Co., Inc, Kenilworth, New Jersey, USA

${ }^{8}$ Department of Clinical Development \& Operations, Pfizer Inc, Collegeville, Pennsylvania, USA

${ }^{9}$ Global Product Development, Pfizer Inc, Groton, Connecticut, USA

Acknowledgements The authors would like to thank the investigators, staff, and participants of the VERTIS CV study (Protocol B1521021), and Christopher P Cannon, Bernard Charbonnel, Philip Jones, Ingrid Adamsons, and Mario Maldonado for their critical review of this work.

Contributors SD-J, REP, DZIC, DKM, JL, RF, JPM, AR, and IG substantially contributed to the conception, design and/or planning of the study. JL, RF, JPM, AR, and IG substantially contributed to the acquisition of data for the study. All authors substantially contributed to the analysis and/or interpretation of the data. SD-J, REP, DZIC, DKM, JL, and IG substantially contributed to the drafting of the manuscript. All authors substantially contributed to the critical review and revision of the manuscript. All authors approved the final version of the manuscript for publication. 
Funding This study was sponsored by Merck Sharp \& Dohme Corp., a subsidiary of Merck \& Co., Inc., Kenilworth, New Jersey, USA, and Pfizer Inc., New York, New York, USA. Editorial support was provided by Shirley Smith of Engage Scientific Solutions (Horsham, UK) and was funded by Merck Sharp \& Dohme Corp., a subsidiary of Merck \& Co., Inc., Kenilworth, New Jersey, USA, and Pfizer Inc., New York, New York, USA.

Competing interests SD-J has led clinical trials for AstraZeneca, Boehringer Ingelheim, and Novo Nordisk; has received consulting fees from AstraZeneca, Bayer, Boehringer Ingelheim, Janssen, Merck \& Co, and Sanofi; has equity interests in Jana Care and Aerami Therapeutics; and serves on the editorial boards of The American Journal of the Medical Sciences, BMJ Open Diabetes Research \& Care, Experimental Biology \& Medicine, and Frontiers in Endocrinology. REP has received grants (directed to his institution) from Hanmi Pharmaceutical Co, Janssen, Metavention, Novo Nordisk, Poxel SA, and Sanofi; has received consulting fees (directed to his institution) from AstraZeneca, Corcept Therapeutics Incorporated, Glytec, Hanmi Pharmaceutical Co, Janssen, Merck \& Co, Mundipharma, Novo Nordisk, Pfizer, Sanofi, Scohia Pharma, and Sun Pharmaceutical Industries; and has received support for attending meetings/travel (directed to his institution or to the travel provider) from AstraZeneca, Glytec, Merck \& Co, Mundipharma, Novo Nordisk, and Pfizer. DZIC has received consulting fees and/or speaking honoraria from AstraZeneca, Bayer, Boehringer Ingelheim, Eli Lilly, Janssen, Merck \& Co, Mitsubishi-Tanabe, Novo Nordisk, Prometic, and Sanofi; and has received operating funds from AstraZeneca, Boehringer Ingelheim, Eli Lilly, Janssen, Merck \& Co, Novo Nordisk, and Sanofi. DKM has received consulting fees from Afimmune, Applied Therapeutics, AstraZeneca, Boehringer Ingelheim, Esperion, Lexicon, Lilly USA, Merck Sharp \& Dohme, Metavant, Novo Nordisk, Pfizer, and Sanofi US; has received honoraria from Boehringer Ingelheim; has received payment for expert testimony from Kirkland \& Ellis on behalf of Boehringer Ingelheim; and has participated on data safety monitoring boards/advisory boards for CSL Behring, AbbVie, Eidos, Otsuka, Arena, and Akebia. FC has received research grants from the Swedish Research Council, Swedish Heart \& Lung Foundation, and King Gustav $V$ and Queen Victoria Foundation; and has received consulting fees from Abbott, AstraZeneca, Bayer, Boehringer Ingelheim, Bristol-Myers Squibb, Merck Sharp \& Dohme, Mundipharma, Novo Nordisk, and Pfizer. JL, AR, and IG are employees of Merck Sharp \& Dohme Corp., a subsidiary of Merck \& Co., Inc., Kenilworth, New Jersey, USA, and may own stock in Merck \& Co., Inc., Kenilworth, New Jersey, USA RF and JPM are employees of, and may own shares/stock options in, Pfizer Inc., New York, New York, USA.

\section{Patient consent for publication Not required.}

Ethics approval The VERTIS CV trial was conducted in compliance with the ethical principles of the Declaration of Helsinki and in compliance with all International Conference on Harmonisation Good Clinical Practice Guidelines. Written informed consent was obtained from all participants, and the final protocol and informed consent documentation were reviewed and approved by the institutional review board or independent ethics committee for each of the 567 investigational centers located across 34 countries.

Provenance and peer review Not commissioned; externally peer reviewed.

Data availability statement Data are available upon reasonable request. Merck Sharp \& Dohme Corp., a subsidiary of Merck \& Co., Inc., Kenilworth, NJ, USA's data sharing policy, including restrictions, is available at http://engagezone.msd. com/ds_documentation.php. Requests for access to the clinical study data can be submitted through the EngageZone site or via email to dataaccess@merck.com.

Supplemental material This content has been supplied by the author(s). It has not been vetted by BMJ Publishing Group Limited (BMJ) and may not have been peer-reviewed. Any opinions or recommendations discussed are solely those of the author(s) and are not endorsed by BMJ. BMJ disclaims all liability and responsibility arising from any reliance placed on the content. Where the content includes any translated material, BMJ does not warrant the accuracy and reliability of the translations (including but not limited to local regulations, clinical guidelines, terminology, drug names and drug dosages), and is not responsible for any error and/or omissions arising from translation and adaptation or otherwise.

Open access This is an open access article distributed in accordance with the Creative Commons Attribution Non Commercial (CC BY-NC 4.0) license, which permits others to distribute, remix, adapt, build upon this work noncommercially, and license their derivative works on different terms, provided the original work is properly cited, appropriate credit is given, any changes made indicated, and the use is non-commercial. See: http://creativecommons.org/ licenses/by-nc/4.0/.
ORCID iDs

Samuel Dagogo-Jack http://orcid.org/0000-0001-5318-9677

Richard E Pratley http://orcid.org/0000-0002-2912-1389

\section{REFERENCES}

1 United States Renal Data System. Chapter 1: CKD in the general population. In: 2020 USRDS annual data report: epidemiology of kidney disease in the United States. Bethesda, MD: National Institutes of Health, National Institute of Diabetes and Digestive and Kidney Diseases, 2020. https://adr.usrds.org/2020/chronic-kidneydisease/1-ckd-in-the-general-population

2 Kibria GMA, Crispen R. Prevalence and trends of chronic kidney disease and its risk factors among US adults: an analysis of NHANES 2003-18. Prev Med Rep 2020;20:101193.

3 Zelnick LR, Weiss NS, Kestenbaum BR, et al. Diabetes and CKD in the United States population, 2009-2014. Clin J Am Soc Nephrol 2017; 12:1984-90.

4 United States Renal Data System. Chapter 4: Cardiovascular disease in patients with CKD. In: 2020 USRDS annual data report: epidemiology of kidney disease in the United States. Bethesda, MD: National Institutes of Health, National Institute of Diabetes and Digestive and Kidney Diseases, 2020. https://adr.usrds.org/2020/ chronic-kidney-disease/4-cardiovascular-disease-in-patients-withckd

5 Jankowski J, Floege J, Fliser D, et al. Cardiovascular disease in chronic kidney disease: pathophysiological insights and therapeutic options. Circulation 2021;143:1157-72.

6 Fioretto P, Zambon A, Rossato M, et al. SGLT2 inhibitors and the diabetic kidney. Diabetes Care 2016;39 Suppl 2:S165-71.

7 Cherney DZ, Odutayo A, Aronson R, et al. Sodium glucose cotransporter-2 inhibition and cardiorenal protection: JACC review topic of the week. J Am Coll Cardiol 2019;74:2511-24.

8 Lopaschuk GD, Verma S. Mechanisms of cardiovascular benefits of sodium glucose co-transporter 2 (SGLT2) inhibitors: a state-of-theart review. JACC Basic Trans/ Sci 2020;5:632-44.

9 American Diabetes Association. 9. Pharmacologic Approaches to Glycemic Treatment: Standards of Medical Care in Diabetes-2020. Diabetes Care 2020;43:S98-110.

10 American Diabetes Association. 11. Microvascular Complications and Foot Care: Standards of Medical Care in Diabetes-2020. Diabetes Care 2020;43:S135-51.

11 Buse JB, Wexler DJ, Tsapas A, et al. 2019 update to: management of hyperglycaemia in type 2 diabetes, 2018. A consensus report by the American Diabetes Association (ADA) and the European Association for the Study of Diabetes (EASD). Diabetologia 2020;63:221-8

12 US Food and Drug Administration. Merck Sharp \& Dohme Corp., Whitehouse Station, NJ, USA. Steglatro ${ }^{\mathrm{TM}}$ (ertugliflozin): prescribing information, 2017. Available: https://www.accessdata.fda.gov/ scripts/cder/daf/index.cfm?event=overview. process\&applno= 209803 [Accessed 1 Jun 2021].

13 European Medicines Agency. MSD (UK) Ltd, Hoddesdon, UK. Steglatro ${ }^{\mathrm{TM}}$ (ertugliflozin): summary of product characteristics, 2018. Available: https://www.ema.europa.eu/en/medicines/human/EPAR/ steglatro [Accessed 1 Jun 2021].

14 Cannon CP, McGuire DK, Pratley R, et al. Design and baseline characteristics of the eValuation of ERTugliflozin efflcacy and Safety CardioVascular outcomes trial (VERTIS-CV). Am Heart $J$ 2018;206:11-23.

15 Cannon CP, Pratley R, Dagogo-Jack S, et al. Cardiovascular outcomes with ertugliflozin in type 2 diabetes. N Engl J Med 2020;383:1425-35

16 Levey AS, Coresh J, Greene T, et al. Using standardized serum creatinine values in the Modification of Diet in Renal Disease study equation for estimating glomerular filtration rate. Ann Intern Med 2006;145:247-54.

17 Fediuk DJ, Nucci G, Dawra VK, et al. End-to-end application of model-informed drug development for ertugliflozin, a novel sodiumglucose cotransporter 2 inhibitor. CPT Pharmacometrics Syst Pharmacol 2021;10:529-42.

18 Liu J, Tarasenko L, Terra SG, et al. Efficacy of ertugliflozin in monotherapy or combination therapy in patients with type 2 diabetes: a pooled analysis of placebo-controlled studies. Diab Vasc Dis Res 2019;16:415-23.

19 Wanner C, Lachin JM, Inzucchi SE, et al. Empagliflozin and clinical outcomes in patients with type 2 diabetes mellitus, established cardiovascular disease, and chronic kidney disease. Circulation 2018;137:119-29. 
20 Neuen BL, Ohkuma T, Neal B, et al. Cardiovascular and renal outcomes with canagliflozin according to baseline kidney function. Circulation 2018;138:1537-50.

21 Fioretto P, Del Prato S, Buse JB, et al. Efficacy and safety of dapagliflozin in patients with type 2 diabetes and moderate renal impairment (chronic kidney disease stage 3A): the DERIVE study. Diabetes Obes Metab 2018;20:2532-40.

22 Grunberger G, Camp S, Johnson J, et al. Ertugliflozin in patients with stage 3 chronic kidney disease and type 2 diabetes mellitus: the VERTIS RENAL randomized study. Diabetes Ther 2018;9:49-66.

23 Cherney DZI, Cooper ME, Tikkanen I, et al. Pooled analysis of Phase III trials indicate contrasting influences of renal function on blood pressure, body weight, and $\mathrm{HbA} 1 \mathrm{c}$ reductions with empagliflozin. Kidney Int 2018;93:231-44.

24 Lautsch D, Alsumali A, McLeod E, et al. Comparative efficacy of dua and single initiation of add-on oral antihyperglycemic agents in type 2 diabetes uncontrolled on metformin alone: a systematic literature review and network meta-analysis. Diabetes Ther 2021;12:389-418.

25 Patel S, Hickman A, Frederich R, et al. Safety of ertugliflozin in patients with type 2 diabetes mellitus: pooled analysis of seven phase 3 randomized controlled trials. Diabetes Ther 2020;11:1347-67.

26 Mosenzon O, Wiviott SD, Cahn A, et al. Effects of dapagliflozin on development and progression of kidney disease in patients with type 2 diabetes: an analysis from the DECLARE-TIMI 58 randomised trial. Lancet Diabetes Endocrinol 2019;7:606-17.
27 Wanner C, Inzucchi SE, Lachin JM, et al. Empagliflozin and progression of kidney disease in type 2 diabetes. $N$ Engl J Med 2016;375:323-34.

28 Dagogo-Jack S. Glycemic control and chronic diabetes complications. In: Umpierrez GE, ed. Therapy for diabetes mellitus and related disorders. 6th ed. Alexandria, VA: American Diabetes Association, 2014: 668-95.

29 Perkovic V, Jardine MJ, Neal B, et al. Canagliflozin and renal outcomes in type 2 diabetes and nephropathy. $N$ Engl J Med 2019;380:2295-306.

30 Heerspink HJL, Stefánsson BV, Correa-Rotter R, et al. Dapagliflozin in patients with chronic kidney disease. N Engl J Med 2020;383:1436-46.

31 McGuire DK, Shih WJ, Cosentino F, et al. Association of SGLT2 inhibitors with cardiovascular and kidney outcomes in patients with type 2 diabetes: a meta-analysis. JAMA Cardiol 2021;6:148-58.

32 Cherney DZI, Charbonnel B, Cosentino F, et al. Effects of ertugliflozin on kidney composite outcomes, renal function and albuminuria in patients with type 2 diabetes mellitus: an analysis from the randomised VERTIS CV trial. Diabetologia 2021;64:1256-67.

33 Cosentino F, Cannon CP, Cherney DZI, et al. Efficacy of ertugliflozin on heart failure-related events in patients with type 2 diabetes mellitus and established atherosclerotic cardiovascular disease: results of the VERTIS CV trial. Circulation 2020;142:2205-15. 